\title{
Patient commentary: Direct access would beat receiving test results with the receptionist's interpretation
}

The authors on both sides of this debate have made an interesting assumption1: that the opposite of patients' direct access is a "traditional model," in which a clinician interprets test results for the patient. To assume that this is the default situation is to miss a vital part of the debate.

In the real world, test results are not just delivered by supportive clinicians, with occasional lapses from that norm; direct access aside, the standard delivery of test results is much more varied than that. I have had results given to me by a general practice receptionist as part of the surgery's normal practice; this included the receptionist's interpretation of what the numbers meant. I have tried to get the results of blood test monitoring and been told by a nurse that these are not given out until the next appointment, with standard waiting time 12 months, by which time the information offered by the test would no longer be useful for self management.

Some clinics send test results by post. I have had devastating diagnostic test results sent in a letter, with context and interpretation so alarmist it made me nearly vomit with fear. One clinic, after doing tests, sends me an appointment card for my next screening. No useful information is offered about what the last set of tests found, but I have come to realise that if my next appointment is in a year's time rather than scheduled more urgently, the results were probably okay. In 25 years of monitoring a serious and complex condition, undergoing investigations for other illnesses, and simply participating in ordinary health promotion screening, these models have been closer to the norm for me than the situation the authors describe.

Of course, other patients may have different experiences. My point is not to say that all patients will agree with me but that there are other standard models besides the one the medical authors are suggesting; they may be less aware of these models precisely because the models do not have the doctor-patient interaction at the centre. A fuller discussion should reflect those receptionists' opinions; those rules about sharing information; those black and white letters that leave the patient unsure where to turn; those contextless appointment cards; and probably much more.

Would I have found it more useful to have had direct access to my results rather than a discussion with my doctor in each of the situations described above? Sometimes yes and sometimes no, depending on my level of knowledge at the time, on the doctor in question, and on the kind of test. Would I have found it more useful to have direct access to my results instead of what actually happened in each of the situations described above? Unequivocally, yes, I would. 